\title{
Importance of Age Factor in Assessing Family History of Coronary Heart Disease
}

\author{
Tomohiro Saito', Toshihito Furukawa², Selichiro Nanri ${ }^{3}$, \\ Ikuo Saito ${ }^{3}$, and Shiro Nagano ${ }^{3}$
}

Age is a major variable to be considered in epidemiology. But age of family members is not considered at all or its consideration seems inadequate in studies on assessing the family history of coronary heart disease as a risk factor. In this report the effects resulting from disregarding age of a family member in such studies were estimated. Age-specific prevalence of the past history of coronary heart disease among 17,926 family members was obtained from the results of a questionnaire survey of 1,716 high school students. From the analysis of the age-specific prevalence by the logistic regression model, an odds ratio was estimated which indicated a risk ratio resulting from a difference in age. The odds ratio obtained was 1.065 (95\% confidence interval: $1.060 \sim 1.071)$. This meant that the odds created by age difference depended on $(1.065)^{n}$, where $n$ was age difference in years. Thus, the effects of disregarding age of family members in assessing the family history of coronary heart disease can be substantial. If the age difference is greater than 5 , its effect expressed by odds becomes larger than 1.370. Some measures to control age of family members need to be taken, especially if the age difference of compared families is not small. J Epidemiol, 1995 ; 5 : 109-112.

family, human genetics, risk, age factors, cardiovascular disease

Age is a major variable to be considered in epidemiology ${ }^{13}$. This necessitates the control for age in most epidemiologic studies including genetic analyses ${ }^{2,3}$. In familial analysis of cancer the importance of controlling age of family members is recognized, and its control seems to be a common practice in some types of cancer ${ }^{4-8)}$. However, this need is neglected or this kind of control is inadequate - not so vigorous as in cancer studies - in many studies on the family history of coronary heart disease whose greatest risk factor is age ${ }^{9)}$ : the age of family members is not considered at all ${ }^{10,11)}$ or control for age seems to be insufficient ${ }^{12,13}$.

Since family history, some claimed ${ }^{14,15)}$, is the most important risk factor for the future development of such diseases, a proper evaluation of family history is crucial in identifying high risk children and youth, one of the major activities in the prevention programs. This study estimates the magnitude of effects resulting from age difference in family members in assessing the family history of coronary heart disease and points out the need for a finer control for age in such studies.

\section{METHODS}

Age-specific prevalence of the past history of coronary heart disease was derived from a questionnaire survey of 1,716 high school students as part of school health programs. The questionnaire contained information on the parents, grandparents and uncles and aunts; the collected data included the present age or age at death, and age at onset, by decade, of angina pectoris and myocardial infarction which had been confirmed by physicians. The questionnaire was filled in at home by parents. Two uncleaunt families were most frequent on both the paternal and maternal side. Thus, data on a total of 17,926 family members were available for calculating the age-specific

Received December 31, 1994 ; accepted June 29, 1995.

'Environmental Epidemiology, National Children's Medical Research Center, Tokyo, Japan.

${ }^{2}$ Division of Biostatistics, Special Reference Laboratories, Inc., Tokyo, Japan.

${ }^{3}$ Health Center, Keio University, Tokyo, Japan.

Address for correspondence: Tomohiro Saito, Environmental Epidemiology, National Children's Medical Research Center, 3-35-31 Taishido Setagaya-ku Tokyo, 154 Japan. 
prevalence of the past history of coronary heart disease.

First, to calculate the age-specific prevalence, the 17,926 members were stratified into 10-year age intervals either by present age or age at death. Second, in each 10-year interval the number of those with a past history of angina pectoris or myocardial infarction and also the number of those who had neither of these disorders were obtained. Third, the age-specific prevalence for each 10-year interval was calculated by the following: [(the number with a past history)/(the number with a past history + the number without a past history)]. Fourth, the following logistic regression model was formulated:

$$
\log (p /(1-p))=\text { intercept }+\beta(\text { decade age })
$$

where $p$ was the 10-year age-specific prevalence and the decade age was the mid-year of the 10-year interval, namely 35 for the interval of 30-39 years, etc. From the logistic model, the parameter $\beta$ was estimated with the numbers of family members in the age intervals as weights in the model. Then, an odds ratio was obtained by an exponent of the estimated $\beta$ together with its $95 \%$ confidence interval by an exponent of $[\beta \pm 1.96 \times$ Standard error of $\beta]$.

The calculations were performed by PC-SAS ${ }^{16)}$ and the logistic model was carried out by the procedure LOGISTIC. The linearity of the explanatory variable was assessed by plotting the logit $\log (p /(1-p))$ against the age scale.

\section{RESULTS}

Figure 1 shows the age-specific prevalence of the past history of coronary heart disease : 10-year prevalence of the presence of a past history of either angina pectoris or myocardial infarction. From the age interval of 30-39 to $60-69$, the prevalence was linear but after age 70 , the increment lessened.

The logit $\log (p /(1-p))$ plotted against the age scale was linear from the mid-year of 30-39 to the mid-year of $60-69$ with a slight decrease in increment in the 70-79, 80-89 and 90-100 intervals. This justified the fitness of the model.

The odds ratio estimated by the logistic regression model was 1.065 . Its $95 \%$ confidence interval was $1.060 \sim 1.071$. Table 1 shows the odds ratios resulting from various age differences calculated by $(1.065)^{n}$, where $n$ was age difference in years.

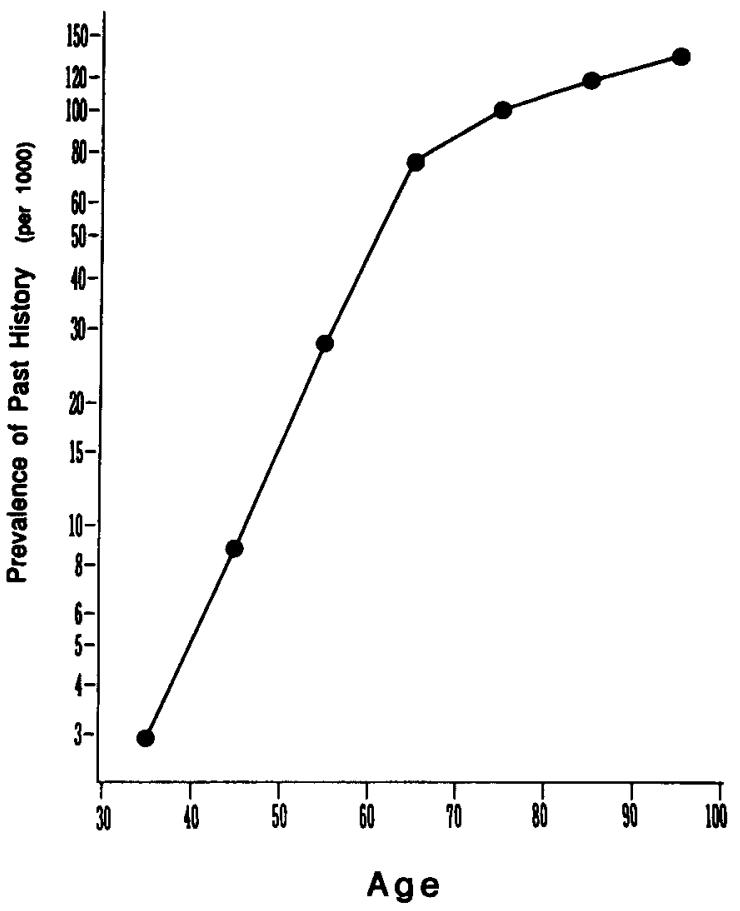

Figure 1. Age-specific prevalence of the past history of coronary heart disease. The mid-years are taken for 10-year intervals on the age axis. The prevalence is on the vertical axis of the log scale.

\section{DISCUSSION}

The results of the analysis showed that the factor of age of family members in assessing the family history of coronary heart disease should not be overlooked. If the age difference is greater than 5 , the odds ratio for the presence of a past history resulting from the age difference can be as large as 1.37 , which is of the same magnitude as the risk ratios of other risk factors in question ${ }^{17,18)}$. An age difference as large as 5 years between parents of different families is not infrequent, judged from the distribution of age of bridegrooms and brides ${ }^{19}$, and of the age of mothers at delivery ${ }^{19}$. Therefore, to assess risk factors among children and youth of adulthood coronary disease from family history, the control for age among families needs to be less than 5 years. The measures of control may be stratification, matching, multivariate analysis or the use of some risk indices which control age ${ }^{20)}$.

Table 1. Odds ratios for the presence of the past history of coronary heart disease resulting from age difference in family members.

\begin{tabular}{lcccccccccc}
\hline Age difference & 1 & 2 & 3 & 4 & 5 & 6 & 7 & 8 & 9 & 10 \\
Odds ratio & 1.07 & 1.13 & 1.21 & 1.29 & 1.37 & 1.46 & 1.55 & 1.65 & 1.76 & 1.88 \\
\hline
\end{tabular}


There are some points to be discussed here before interpreting the results. First, misclassification in the family history: whether information on present age, age at death, the past history of coronary heart disease and age at its onset expressed by decade was reliable. The questionnaire was filled in at home by parents. It is unlikely that people in their 40 s and 50 s, namely parents in the questionnaire, were mistaken in answering present age and age at death of their brothers, sisters and parents. The past history of coronary heart disease and its onset by decade age should be quite accurate on their brothers and sisters - uncles and aunts in the questionnaire - as they were in the same age range, and members of average families contact and meet each other regularly once or twice a year in Japan. The grandparents in the questionnaire were mostly in their 60 s and 70 s by present age or age at death. Their sons and daughters - parents in the questionnaire - most likely know the past history of such serious disease as angina pectoris or myocardial infarction, and information on its onset should be reliable if expressed by decade age.

In another study we examined the validity of a family history questionnaire, administered twice with a 4 month interval, on the past history of atherosclerotic diseases among parents and grandparents of children ${ }^{21)}$. The proportions of contradicting answers - namely an interchange between the presence and absence of a past history - between the two surveys among such family members were low on the past history of angina pectoris and myocardial infarction : $1.0 \%$ and $0.8 \%$ respectively. Thus, precision was shown to be good in obtaining data on such disorders. On the other hand the inaccuracy of such information, namely a discrepancy between answers and true evidence, is difficult to confirm. But it may not exceed $5 \%$. Within this range of inaccuracy, the estimated odds ratio by the logistic regression model in this paper changes very little.

Another point to be discussed is period and cohort effects. The prevalence of the past history of coronary heart disease at the same age may differ between different periods. If the incidence of coronary heart disease has been increasing, the prevalence of the past history should become higher among present-day cohorts than past cohorts of the same age. This leads to a less steep slope in age-specific prevalence of the past history than that shown in Figure 1. In this case the estimated odds ratio in this study should be interpreted as an underestimated one when the results of this study are applied to other populations of different periods. However, age-specific mortality from coronary heart disease in Japan has decreased slightly in the past 4 decades $^{22}$. Therefore, it is not likely that age-specific prevalence of the past history changed so much as to endanger the results of this study.

It is noted here that the concept of prevalence of the past history of a certain disease is close to that of cumulative incidence, the proportion of a fixed population that becomes diseased in a stated period of time ${ }^{23)}$. A difference between them is that cumulative incidence is premised on the assumption of no competing risks of death $^{23}$. In prevalence of the past history, however, persons who died from other diseases are included in the denominator and the presence of the past history of a disease concerned in such persons is usually counted in the numerator. Within the age range where death rates from all causes are not high, both rates should be close. Since data on prevalence of the past history are rarely available, they can be substituted by cumulative incidence ${ }^{24)}$. Cumulative incidence is derived from incidence. It approximates $\Sigma I_{i} \cdot \Delta t_{i}$ where $I_{i}$ denotes the age-specific incidence rate at time $\mathrm{i}$ and $\Delta t_{i}$ denotes time or age ${ }^{23}$.

It follows from the above two relations that the prevalence of the past history is regarded as a transformed index of incidence. This leads to the following: in countries where the incidence of coronary heart disease is high, the prevalence of its past history among family members is also high.

In such countries as the U.S.A. and other Western countries where the incidence of coronary heart disease and its mortality are higher than those in Japan, their increase with age is steeper than that in Japan ${ }^{9,25)}$. Similar analyses in such countries should yield larger odds ratios than the odds ratio estimated in this study. Control for age in assessing family history should be requested more urgently, and a finer control for age will be requested in these countries.

The importance of an age factor in assessing family history needs to be borne in mind also in clinical practice, in which family history is almost always taken. The age of family members needs to be considered if family history is taken as a risk factor for a patient.

The neglect of control for age of family members is not uncommon in evaluating the family history of other diseases with a steep increase in age-specific incidence including hypertension ${ }^{26,27)}$, diabetes ${ }^{26,28)}$ and stroke $^{26,29)}$. The necessity for the control for age in family history assessment needs to be widely recognized.

\section{ACKNOWLEDGEMENTS}

Supported in part by a grant-in-aid "Research on the establishment of healthy lifestyle from childhood" from the Ministry of Health and Welfare, Japan. The authors thank Ms. R. Fallon for technical advice in writing.

\section{REFERENCES}

1. MacMahon B, Pugh TF. Epidemiology - principles and methods. Little, Brown and Company, Boston, 1970 ; 103136. 
2. Cavalli-Sforza LL, Bodmer WF. The Genetics of Human Populations. WH Freeman and Company, San Francisco, 1971: 304-307.

3. Saito T. Evaluation of segregation ratio in Wilson's disease. J Med Genet 1983; 20: 271-275.

4. Macklin MT. Comparison of the number of breast-cancer deaths observed in relatives of breast-cancer patients, and the number expected on the basis of mortality rates. J Natl Cancer Inst $1959 ; 22$ : 927-951.

5. Anderson DE. Genetic study of breast cancer : identification of a high risk group. Cancer $1974 ; 34: 1090$ 1097.

6. Weber BL, Garber JE. Family history and breast cancer : probabilities and possibilities. JAMA 1993;270:16021603.

7. Bain C, Speizer FE, Rosner B, Belanger C, Hennekens CH. Family history of breast cancer as a risk indicator for the disease. Am J Epidemiol 1980; 111: 301-318.

8. Ooi WL, Elston RC, Chen VW, Bailey-Wilson JE, Rothchild $H$. Increased familial risk for lung cancer. J Natl Cancer Inst $1986 ; 76: 217-222$.

9. WHO Expert Committee. Prevention of coronary heart disease. World Health Organization Tech Rep Series No. $678,1982$.

10. Shear CL, Burke GL, Freedman DS, Berenson GS. Value of childhood blood pressure measurements and family history in predicting future blood pressure status: results from 8 years of follow-up in the Bogalusa Heart Study. Pediatrics 1986 ; 77 : 862-869.

11. Murata M, Yamazaki K. The system for preventing adult vascular diseases in infancy and school age with emphasis on follow-up individuals and populations. In : Ookuni M, ed. The Study for the Prevention of Adult Vascular Diseases from Childhood. Report of the Study Group in Psychological and Health Disturbances, Ministry of Health and Welfare, Japan. 1993: 93-101.

12. Nora JJ. Identifying the child at risk for coronary disease as an adult : a strategy for prevention. J Pediatr 1980; 97 : 706-714.

13. NCEP Expert Panel on Blood Cholesterol Levels in Children and Adolescents. National Cholesterol Education Program (NCEP) : highlights of the report of the expert panel on blood cholesterol levels in children and adolescents. Pediatrics 1992; 89 : 495-501.

14. Motulsky AG. Genetic research in coronary heart disease. In : Rao DC, Elston RC, Kuller LH, Feinleib M, Carter C, Havlik R, eds. Genetic epidemiology of coronary heart disease. Alan R. Liss, Inc., New York, 1984: 541-548.
15. Goldbourt U, Neufeld N. Genetic aspects of arteriosclerosis. Arteriosclerosis $1986 ; 6: 357-377$.

16. SAS Institute Inc. PC-SAS, SAS/BASE, SAS/STAT, SAS/GRAPH software. Version 6, Cary, NC, USA.

17. Klag MJ, Ford DE, Mead LA, He J, Whelton PK, Liang KY, Levine DM. Serum cholesterol in young men and subsequent cardiovascular disease. N Engl J Med 1993; 328 : 313-318.

18. Kaplan GA, Salonen JT. Socioeconomic conditions in childhood and ischaemic heart disease during middle age. Br Med J 1990; 301 : 1121-1123.

19. United Nations. Marriages by age of bridegroom and by age of bride. Live births by age of mother, sex and urban/ rural residence. In : Demographic Yearbook 1992, United Nations, New York, 1994: 307-317, 342-408.

20. Kobberling J. Studies on the genetic heterogeneity of diabetes mellitus. Diabetologia 1971; 7 : 46-49.

21. Saito T, Yamagami K, Kagamimori S. Family history assessment in the Toyama Study: validity. In : Fukuwatari Y, ed. The Study for the Establishment of Healthy Lifestyle from Childhood. Report of the Study Group in Psychological and Health Disturbances, Ministry of Health and Welfare, Japan. 1994 : $271-277$.

22. Statistics and Information Department, Ministry of Health and Welfare. Death rates by sex, age and causes of death. In : Vital Statistics of Japan. Tokyo: Ministry's Secretariat, Ministry of Health and Welfare, 1950-1993.

23. Rothman KJ. Modern Epidemiology. Little, Brown and Company, Boston, 1986 : 23-34.

24. Hunt SC, Williams RR, Barlow GK. A comparison of positive family history definitions for defining risk of future disease. J Chron Dis 1986; $39: 809-821$.

25. Uemura K, Pisa $Z$. Trends in cardiovascular disease mortality in industrialized countries since 1950 . World Health Stat Q 1988; 41: 155-178.

26. Burke GL, Savage PJ, Sprafka JM, et al. Relation of risk factor levels in young adulthood to parental history of disease. The CARDIA study. Circulation 1991; 84: 1176-1187.

27. Lauer RM, Clarke WR. Childhood risk factors for high adult blood pressure: The Muscatine Study. Pediatrics $1989 ; 84: 633-641$.

28. Cheta D, Dumitrescu C, Georgescu M, et al. A study on the types of diabetes mellitus in first degree relatives of diabetic patients. Diabete \& Metab 1990; $16: 11-15$.

29. Graffagnino C, Gasecki AP, Doig GS, Hachimski VC. The importance of family history in cerebrovascular disease. Stroke $1994 ; 25$ : 1599-1604. 